\title{
Heterologous expression of the AtDREB1A gene in tomato confers tolerance to chilling stress
}

\author{
S.G. KARKUTE ${ }^{1}$, R. KRISHNA ${ }^{1}$, W.A. ANSARI ${ }^{1}$, B. SINGH $^{1}$, P.M. SINGH $^{1}$, M. SINGH ${ }^{2}$, \\ and A.K. SINGH ${ }^{1 *}$
}

ICAR-Indian Institute of Vegetable Research, Varanasi - 221305, Uttar Pradesh, India ${ }^{1}$

ICAR-Directorate of Onion and Garlic Research, Pune - 410505, Maharashtra, India ${ }^{2}$

\begin{abstract}
Tomato is highly sensitive to chilling stress $\left(0-12{ }^{\circ} \mathrm{C}\right)$ which severely affects plant growth and development. Transgenic tomato plants expressing the AtDREB1A gene under the control of the rd29A promoter were evaluated for its tolerance to chilling stress by exposing them to $4{ }^{\circ} \mathrm{C}$ for $5 \mathrm{~d}$. The cold stress caused an increase in production of reactive oxygen species, however, transgenic plants had an effective antioxidant system due to an enhanced synthesis of catalase (CAT), superoxide dismutase (SOD), and ascorbate and so the reduced content of hydrogen peroxide and superoxide anions. Transgenic plants showed a slightly less reduction of chlorophyll and carotenoid content compared to wild-type plants. Similarly, a higher relative water content and a less electrolyte leakage were observed in transgenic plants. Accumulation of osmoprotectants, like proline and soluble sugars, helped transgenic plants to maintain a proper osmotic balance under the cold stress. Stress-responsive genes pyrroline-5-carboxylate synthase, CAT, SOD, and lipid peroxidase showed enhanced expressions under the cold stress in transgenic plants compared to wild-type plants. A recurrent exposure to the cold stress at the reproductive stage showed even higher expressions of these genes as compared to plants exposed to the cold stress for the first time. Thus, transgenic plants showed a better adaptation to the cold stress than non-transgenic plants by acquiring the stress memory of the stress experienced at the seedling stage.
\end{abstract}

Additional key words: catalase, proline, Solanum lycopersicum, stress memory, stress-responsive genes, superoxide dismutase, transgenic plants.

\section{Introduction}

Tomato (Solanum lycopersicum) is one of the most important vegetable crops worldwide. Abiotic stresses like drought, heat, cold, nutrient deficiency, and excess of salts or toxic metals are the major environmental factors affecting tomato production worldwide. Adverse effects of these environmental stresses are intensified by the climate change (Fedoroff et al. 2010). Tomato is highly sensitive to chilling stress and its growth is adversely affected by temperatures below $12{ }^{\circ} \mathrm{C}$. Tomato plants are negatively affected by cold stress resulting in lower germination of seeds, a decreased photosynthesis and respiration, defects in water status and development, poor pollen germination, etc. (Weiss and Egea-Cortines 2009). The fruits at the ripening stage suffer from a chilling injury, which reduces the marketable fruit yield.
Plants experience cold stress in two forms, freezing $\left(<0{ }^{\circ} \mathrm{C}\right)$ and chilling $\left(0-15{ }^{\circ} \mathrm{C}\right)$, and they exhibit differential responses to these stresses. Plants exposed to chilling often enhance expressions of cold-responsive genes resulting in various biochemical and physiological changes (Chinnusamy et al. 2006). Among others, it leads to an altered lipid composition of membranes and accumulation of sugars and osmolytes (YamaguchiShinozaki and Shinozaki 2006).

Plants respond to environmental stresses by triggering an array of morphological, physiological, biochemical, and molecular processes enabling the plants to survive and reproduce under adverse conditions (Akhtar et al. 2012). Abiotic stress leads in plants to the production of reactive oxygen species (ROS), which are key signal

Submitted 26 April 2018, last revision 9 October 2018, accepted 11 October 2018.

Abbreviations: AsA - ascorbic acid; CAT - catalase; DREB - dehydration responsive element binding; EL - electrolyte leakage; LPO - lipid peroxidase; P5CS - pyrroline-5-carboxylate synthase; rd29A - responsive to dehydration 29A; ROS - reactive oxygen species; RT - reverse transcription; RWC - relative water content; SOD - superoxide dismutase; TSS - total soluble sugars.

Acknowledgement: This study was funded by the National Project on Transgenic Crops (NPTC), Indian Council of Agricultural Research, New Delhi, India.

* Corresponding author; fax: (+91) 5443229007, e-mail: achuits@gmail.com 
molecules regulating various pathways during plant acclimation to a stress. At the same time, it creates an oxidative stress and damages cell membranes, DNA, RNA, and protein molecules. Therefore, managing these ROS is an important activity in plants during stress conditions. Transcription factors regulate numerous downstream genes in response to particular stress. Particularly under cold stress, C-repeat binding factor/dehydration responsive element binding $(C B F /$ $D R E B)$ transcription factors play an important role in controling the expression of cold-responsive $(C O R)$ genes in Arabidopsis (Gilmour et al. 1998). Transgenic plants overexpressing Arabidopsis CBF1 show induction of $C O R$ genes and an increased tolerance to cold stress (Jaglo-Ottosen et al. 1998). Since then, several reports have shown that the over-expression of a $C B F / D R E B 1$ transcription factor enhances tolerance to low temperatures in transgenic tobacco (Liu et al. 2015), Arabidopsis, and rice (Zong et al. 2016) plants.

\section{Materials and methods}

Plants and treatments: Tomato (Solanum lycopersicum Mill.) wild-type plants of cv. Kashi Vishesh (H86) and transgenic plants with AtDREB1A obtained from Indian Institute of Vegetable Research, ICAR, were grown in pots filled with a sterile soil and compost in a glasshouse at a temperature of about $25^{\circ} \mathrm{C}$. For cold treatment, one set of 25 -d-old seedlings of both transgenic and wild-type plants (10 plants each) were subjected to $4{ }^{\circ} \mathrm{C}$ for $5 \mathrm{~d}$ in a growth chamber (Blue Star, New Delhi, India) at a 12-h photoperiod, an irradiance of $150 \mu \mathrm{mol} \mathrm{m} \mathrm{m}^{-2} \mathrm{~s}^{-1}$, and an air humidity of $50-60 \%$ and then transferred back to the glasshouse. Controls were grown in the glasshouse at optimal conditions. After one month, all the stressed and nonstressed plants were exposed to $4{ }^{\circ} \mathrm{C}$ for $30 \mathrm{~min}$ to check the effect of earlier cold stress on responses of plants to this subsequent stress. Leaf samples were collected from all the treated and non-treated plants in 3 biological replicates for biochemical and molecular analyses. The collected samples were quickly frozen in liquid nitrogen and stored at $-80{ }^{\circ} \mathrm{C}$.

Analysis of biochemical, physiological and morphological parameters: In control plants and plants subjected to $4{ }^{\circ} \mathrm{C}$ for $5 \mathrm{~d}$, relative water content (RWC) and electrolyte leakage (EL) were measured according to Khare et al. (2010). Proline content in leaves was measured by the method of Bates et al. (1973) comparing the data to a standard curve of proline ( 5 to $100 \mu \mathrm{g} \mathrm{cm}^{-3}$ ). The method of Heath and Packer (1968) was used for measuring lipid peroxidation products expressed as total malondialdehyde (MDA) content. Coefficient of absorption was $155 \mathrm{mM}^{-1} \mathrm{~cm}^{-1}$. Hydrogen peroxide $\left(\mathrm{H}_{2} \mathrm{O}_{2}\right)$ content of leaf samples was estimated by the method of Jana
Transgenic tomato cv. Kashi Vishesh expressing AtDREB1A under the control of the responsive to dehydration 29A (rd29A) promoter were developed earlier in our lab (Rai et al. 2012, 2013). The objective of the present study was to investigate, by analyzing physiological, biochemical, and molecular changes of transgenic tomato under cold stress, whether an inducible expression of AtDREBIA enhances cold tolerance in those plants. Further, we have studied whether transgenic plants remember cold stress experienced at the early stage of growth and show a response in a better way to subsequent cold stress at the flowering stage compared to non-transgenic plants because it has been reported earlier that plants can remember stress in order to protect themselves from subsequent exposure to stress conditions (Wibowo et al. 2016). Wibowo et al. (2016) have further shown that plants could pass on the stress memory to their offspring in the form of change in methylation pattern of genomic DNA.

and Choudhury (1981). The total soluble sugars (TSS) were estimated by following the method given by DuBois et al. (1956). The method described by Chaitanya and Naithani (1994) was used for measuring the rate of superoxide anion $\left(\mathrm{O}_{2}{ }^{\bullet-}\right)$ production. Further, the total ascorbic acid (AsA) and reduced AsA were extracted and quantified using the assay of Law et al. (1983). The protocol of Mishra and Fridovich (1972) was followed for superoxide dismutase (SOD) assay. The similar method described by Aebi (1984) was used for catalase (CAT) specific activity. The amounts of chlorophylls and carotenoids were determined according to the method of Lichtenthaler and Buschmann (2001). In addition, root shoot lengths, fresh and dry masses of shoots, roots, and leaves were recorded. For dry mass determination, these plant parts were dried in an oven at $80{ }^{\circ} \mathrm{C}$ for $48 \mathrm{~h}$.

Iolation of RNA and reverse transcription (RT) quantitative (q) PCR: Five stress associated genes AtDREB1A, CAT, SOD, pyrroline-5-carboxylate synthase (P5CS), and lipid peroxidase (LPO) were selected, and their nucleotide sequences were retrieved from the NCBI database (www.ncbi.nlm.nih.gov). The Primer3 v. 0.9 software (Rozen and Skaletsky 1998) was used to design primers for PCR. Total RNA was extracted from tomato leaf samples using a TRI reagent (Ambion, CA, USA) along with RNAase free DNAase treatment (Qiagen, Hilden, Germany). One microgramme of total RNA was used for first strand cDNA synthesis in $20 \mathrm{~mm}^{3}$ of reaction volume. A first strand cDNA synthesis kit (Bio-Rad, Hercules, USA) was used, and IQ SYBR Green Supermix (Bio-Rad) was used for RT-qPCR on an $i Q 5$ thermal cycler (Bio-Rad) with the $i Q 5$ software. The actin gene was used as an internal control. Nucleotide sequences of gene-specific 
primers and actin are given in Table 1 Suppl. The RT-qPCR was performed by following the manufacturer's protocol. The cDNA $\left(5 \mathrm{~mm}^{3}\right)$ was used for PCR reaction and temperature cycles were: an initial incubation at $95{ }^{\circ} \mathrm{C}$ for $1 \mathrm{~min}$, then 35 cycles of $95^{\circ} \mathrm{C}$ for $30 \mathrm{~s}$, at $55-60^{\circ} \mathrm{C}$ (a primer specific $\mathrm{Tm}$ ) for $30 \mathrm{~s}, 72{ }^{\circ} \mathrm{C}$ for $40 \mathrm{~s}$, and a final extension at $72{ }^{\circ} \mathrm{C}$ for $5 \mathrm{~min}$. The $2^{-\triangle \Delta C T}$ method (Livak and Schmittgen 2001) was utilized for detecting relative gene expressions.

\section{Results}

To study the effect of inducible expression of AtDREB1A on RWC and EL, plants were subjected to chilling stress of $4{ }^{\circ} \mathrm{C}$ for $5 \mathrm{~d}$. After exposure to the cold stress, wildtype plants showed wilting, whereas transgenic plants exhibited a normal growth and development. No large differences were observed in RWC of transgenic and wild-type plants under optimal growth conditions, however, a higher RWC was noticed in transgenic plants exposed to the chilling stress as compared to wild-type plants making them more tolerant to the low-temperature
Statistical analyses: For statistical analysis, means and standard errors were calculated. One way analysis of variance $(A N O V A)$ was used for data analysis at $\alpha=0.05$. The analysis was conducted using the SPSS v. 16.0 software (SPSS, Chicago, USA). To compare mean values, the Duncan multiple range test was performed.

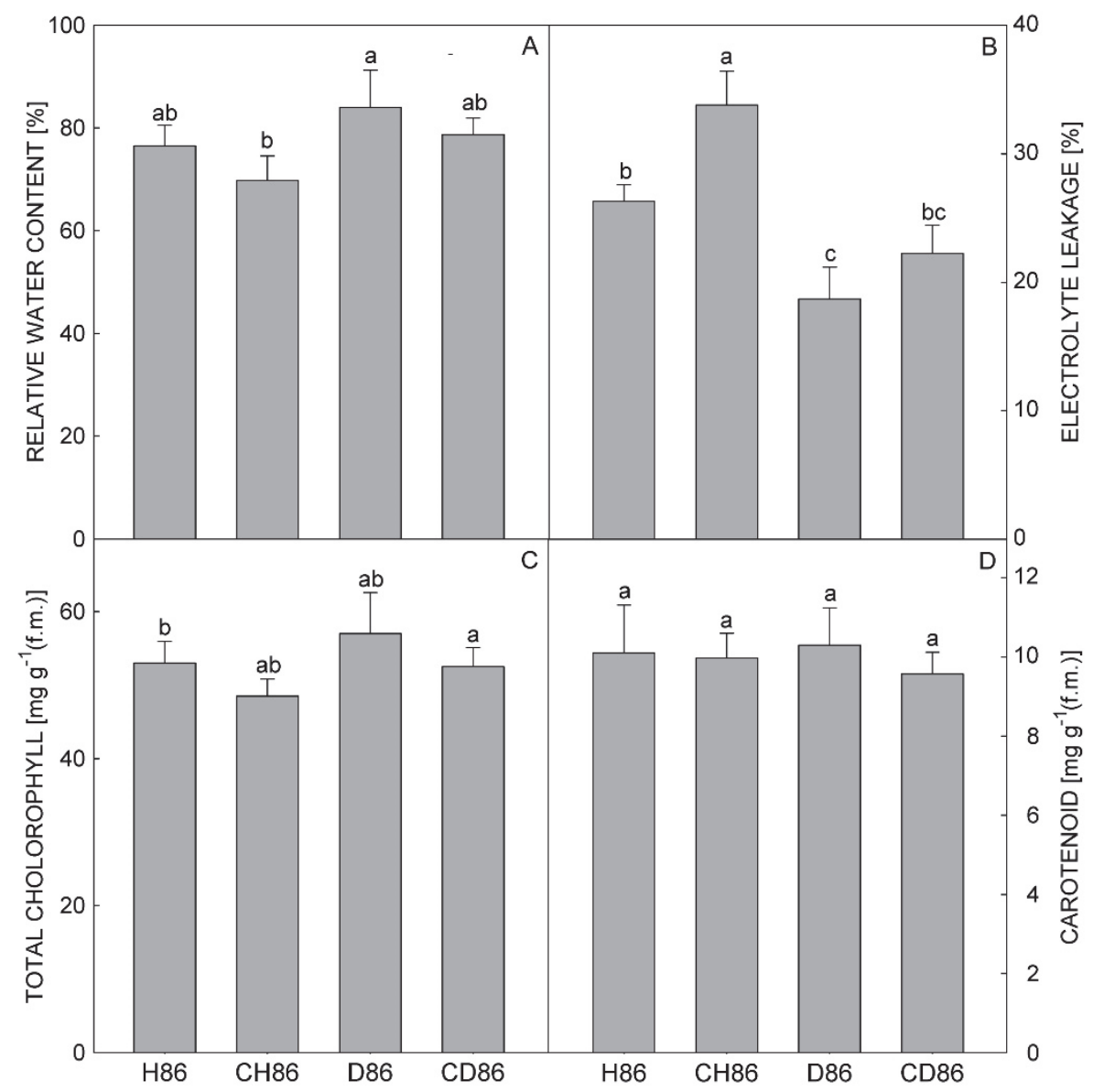

Fig. 1. Effect of cold stress $\left(4^{\circ} \mathrm{C}\right.$ for $\left.5 \mathrm{~d}\right)$ on relative water content $(A)$, electrolyte leakage $(B)$, total chlorophyll content $(C)$, and carotenoid content $(D)$ in wild-type (H86) and transgenic (D86) tomato plants. Means \pm SEs, $n=3$, values followed by the same letter are not significantly different $(P>0.05)$ according to the Duncan multiple range test. CH86 - wild type cold treated plants, CD86 - transgenic cold treated plants. 
Total chlorophyll content was slightly reduced in both transgenic and wild-type plants after the cold stress. However, the reduction was lower in transgenic plants (Fig. 1C). Carotenoid content was not significantly affected (Fig. 1D). Further, it was observed that the accumulation of proline increased under the cold stress in both transgenic and wild-type plants, however, the change was more profound in wild-type plants. Although the increase in proline content under the cold stress was larger in wild-type plants, overall proline content was higher in transgenic plants in both control and stress conditions (Fig. 2A). The cold stress enhanced the accumulation of TSS and so helped to retain water in cells. Transgenic plants exhibited a higher content of TSS even under optimal conditions compared to wild-type plants. Exposure to the cold stress further enhanced total soluble sugar content and a higher change was observed in wild-type $(18.21 \%)$ than transgenic plants $(15.06 \%$, Fig. $2 B)$.

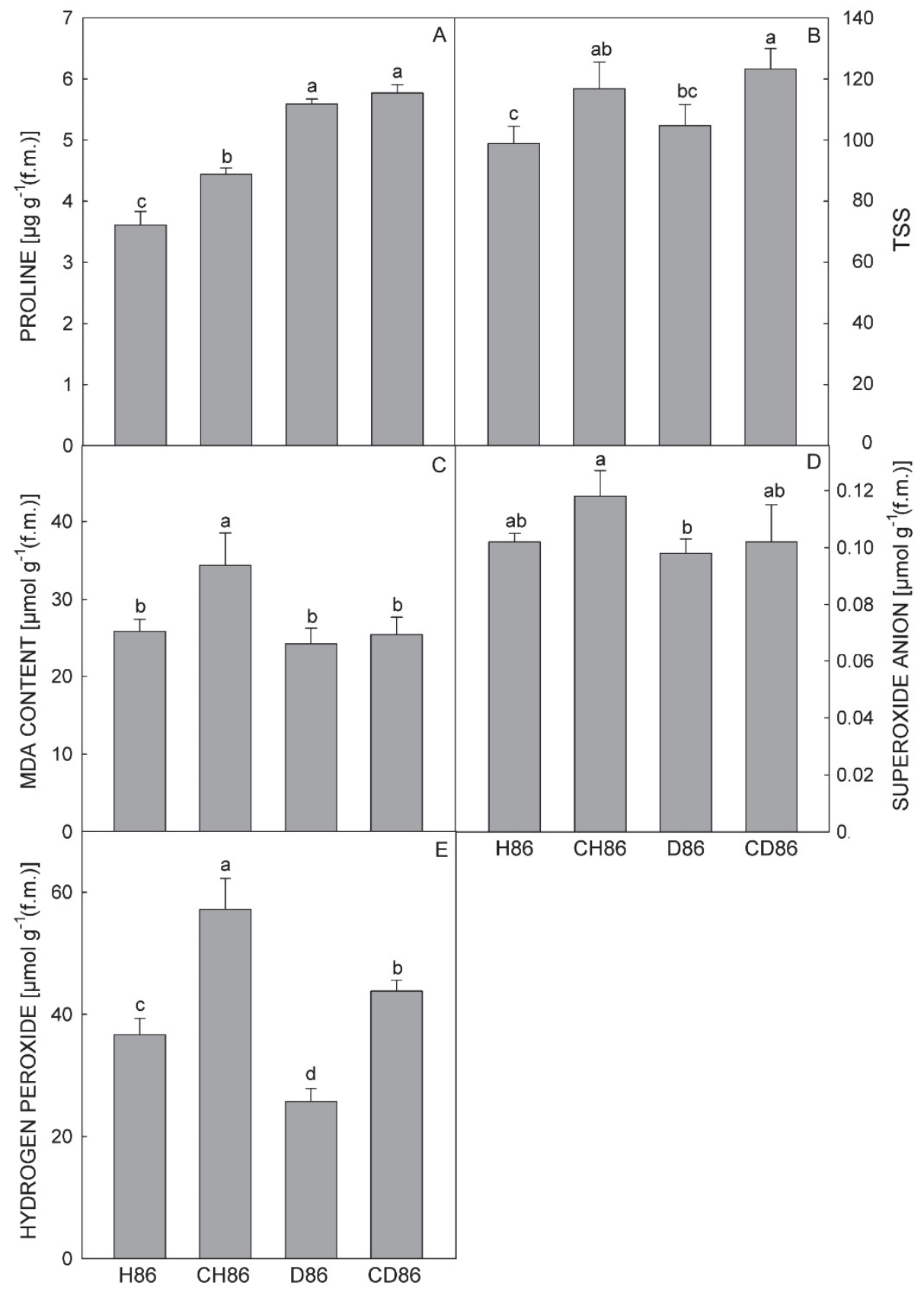

Fig. 2. Effect of cold stress $\left(4{ }^{\circ} \mathrm{C}\right.$ for $\left.5 \mathrm{~d}\right)$ on proline content $(A)$, total soluble sugar (TSS) content $(B)$, malondialdehyde (MDA) content $(C)$, superoxide anion $(D)$, and hydrogen peroxide $(E)$ in wild-type (H86) and transgenic (D86) tomato plants; Means \pm SEs, $n=3$, values followed by the same letter are not significantly different $(P>0.05)$ according to the Duncan multiple range test. CH86 - wild type cold treated plants, CD86 - transgenic cold treated plants. 


\section{S,G, KARKUTE et al.}

The cold stress significantly increased MDA content in plants, however, less $(21.35 \%)$ in transgenic plants compared to wild-type plants (33.07\%; Fig. 2C). Also, an increase in superoxide anion content was found under the cold in both transgenic and wild-type lines. This increase was $15.68 \%$ in wild-type plants and only $4.08 \%$ in transgenic plants (Fig. 2D). This indicates that cold exposed transgenic plants experienced a lower chillinginduced oxidative stress compared to wild-type plants. Hydrogen peroxide content was enhanced in both types of plants under the cold stress, however, a larger increase of $70.34 \%$ in $\mathrm{H}_{2} \mathrm{O}_{2}$ content was observed in transgenic plants (Fig. 2E). However, transgenic plants showed a lower $\mathrm{H}_{2} \mathrm{O}_{2}$ content under control conditions. Although the increase in $\mathrm{H}_{2} \mathrm{O}_{2}$ content was higher in transgenic plants under the cold stress, the overall amount of $\mathrm{H}_{2} \mathrm{O}_{2}$ was still lower in wild-type plants. Increases in activities of SOD and CAT, were noticed in both transgenic and wild-type plants under the cold stress (Fig. $3 A, B$ ). The increases in activities of SOD and CAT were high (29.49 and $21.34 \%$, respectively) only in transgenic plants. Measurement of AsA under the cold stress reveals that reduced AsA and total AsA content increased under the cold stress in both transgenic and wild-type plants (Fig. $3 C, D)$. The total AsA content significantly increased only in transgenic plants helping them effectively scavenge ROS.

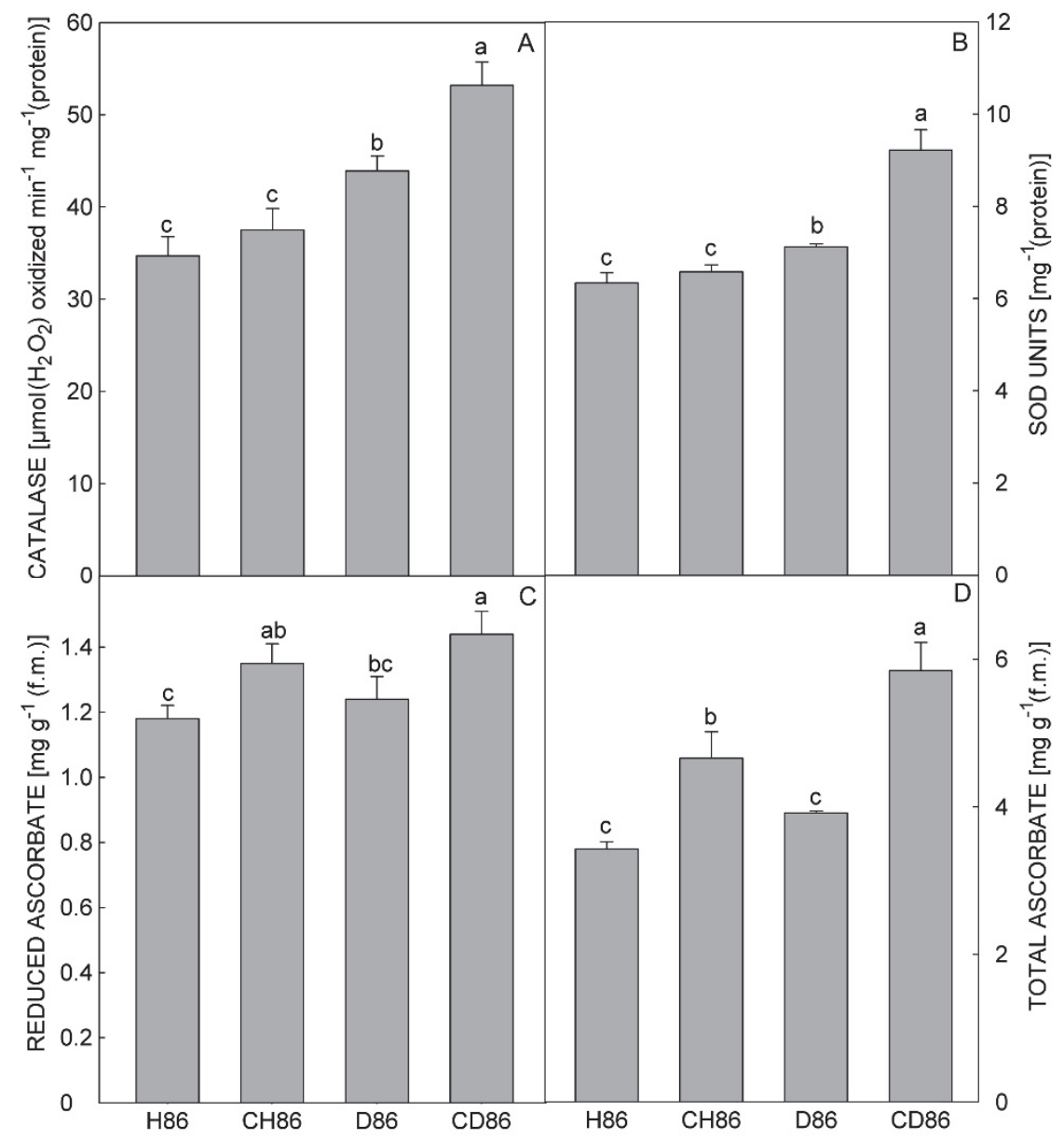

Fig. 3. Effect of cold stress $\left(4{ }^{\circ} \mathrm{C}\right.$ for $\left.5 \mathrm{~d}\right)$ on activities of catalase $(A)$ and superoxide dismutase $(B)$ and on content of reduced ascorbate $(C)$ and total ascorbate $(D)$ in wild-type (H86) and transgenic (D86) tomato plants. Means \pm SEs, $n=3$, values followed by the same letter are not significantly different $(P>0.05)$ according to the Duncan multiple range test. CH86 - wild type cold treated plants, CD86 - transgenic cold treated plants.

In general, cold stress affects growth of plants. Surprisingly, shoot length increased in transgenic plants. Although the increased root length was observed in both wild-type and transgenic plants under the cold stress, the increase was higher in wild-type plants. The cold stress also affected the dry to the fresh mass ratio of shoots and roots with a more reduction in transgenic plants compared to wild-type plants (Table 2 Suppl.)

To investigate changes in gene transcriptions under the cold stress in AtDREBIA expressing transgenic plants, expressions of $A t D R E B 1 A$ and genes involved in osmotic adjustment (P5CS) and antioxidant defense $(C A T, S O D$, and $L P O)$ were analyzed by RT-qPCR. The gene expression analysis shows that $S O D, C A T$, and $L P O$ 
were up-regulated in both types of plants after exposure to the cold stress (Fig. 4A-C). However, expressions in transgenic plants were significantly higher than in wildtype plants at both optimal and stress conditions except in case of $L P O$. Transgenic plants showed enhanced expressions of $S O D$ and $C A T$ genes even under optimal conditions compared to wild-types. The expression of LPO was slightly induced in both plant types after cold stress exposure, although it is lower in transgenic plants. The expression of the P5CS gene was significantly induced in transgenic tomato plants under the cold stress (Fig. 4D), which explains an increased proline content in transgenic plants under the cold stress. Finally, the expression of AtDREB1A increased in transgenic plants under the cold stress (Fig. 5E).

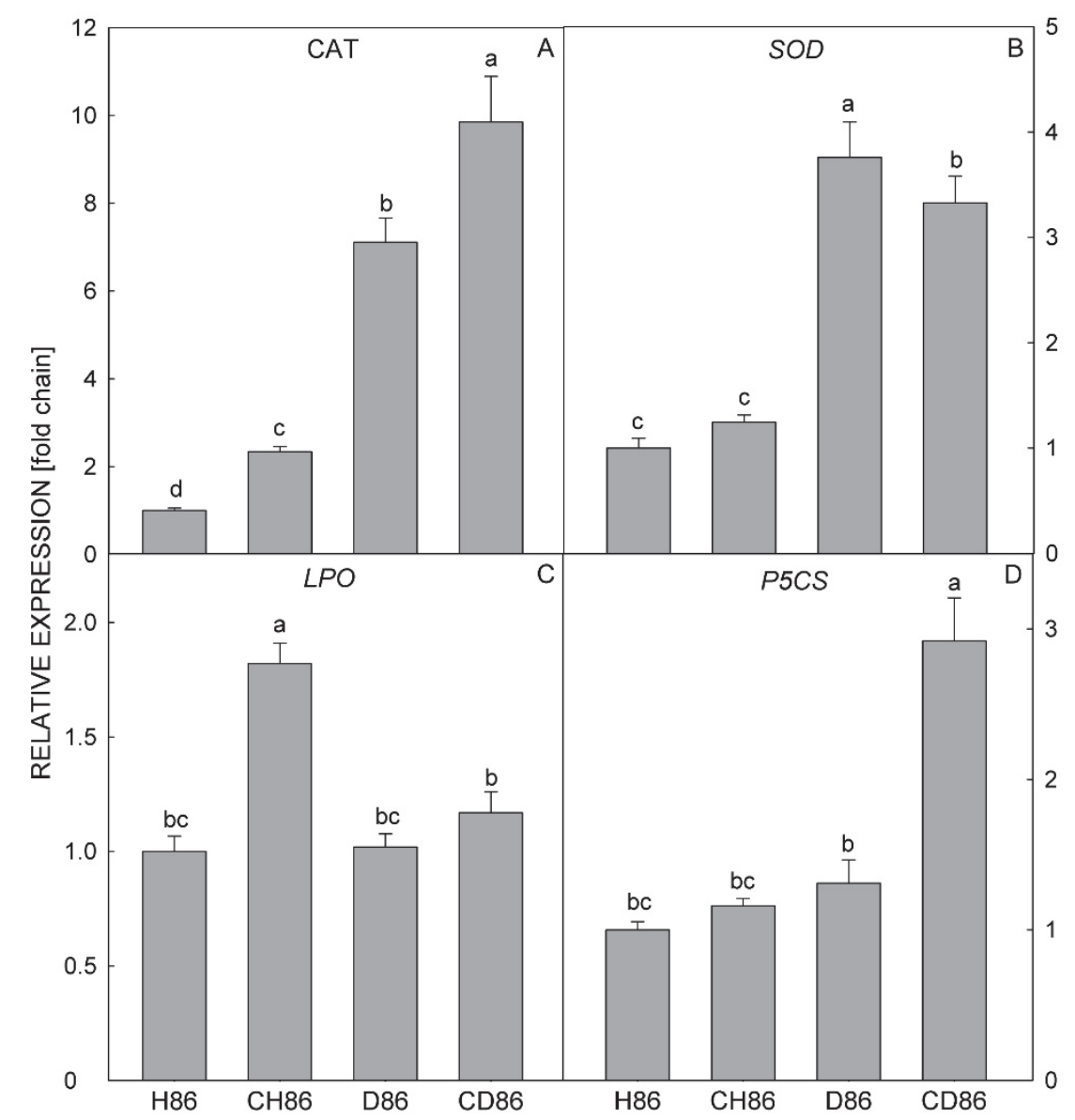

Fig. 4. Effect of cold stress $\left(4^{\circ} \mathrm{C}\right.$ for $\left.5 \mathrm{~d}\right)$ on expression of cold responsive genes $C A T(A), S O D(\mathrm{~B}), \operatorname{LPO}(C)$, and $P 5 C S(D)$ in wildtype (H86) and transgenic (D86) plants. Means \pm SEs, $n=3$, values followed by the same letter are not significantly different $(P>0.05)$ according to the Duncan multiple range test. CH86 - wild type cold treated plants, CD86 - transgenic cold treated plants.

Further, the response of plants to the subsequent cold stress was studied by analyzing the expressions of $A t D R E B 1 A, P 5 C S, S O D, C A T$, and $L P O$ genes. It was observed that both transgenic and wild-type plants which had experienced the cold stress at the initial growth stage showed higher expressions of all these genes compared to plants which had experienced the cold stress for the first time (Fig. 5). The subsequent cold stress to plants at the

\section{Discussion}

Tomato plants are highly sensitive to low-temperature stress. Traditional breeding for developing cold tolerant genotypes in tomato is limited by the availability of reproductive stage significantly induced the expressions of stress-responsive genes within $30 \mathrm{~min}$ of the stress indicating a quick response of plants. In contrast, plants which had experienced the cold stress for the first time revealed a slower response to the cold stress. Further, transgenic plants showed a better response to the subsequent cold stress in comparison to non-transgenic plants. genetic resource of cold tolerance in crossable species and, therefore, modern biotechnological approaches have been utilized to understand the mechanism of cold stress 
tolerance in plants. Plants respond to cold stress in a complex and integrated way. This is regulated by endogenous cold responsive element-binding factor pathway, which induces an array of physiological and biochemical modifications at cellular, tissue, and organ levels in plants (Pino et al. 2008). The adaptability of plants to cold stress is linked to certain signal transduction pathways, which ensure the induction of several cold-regulated genes leading to stress tolerance in plants (Janska et al. 2011). Reactive oxygen species generated due to stress play an important role in this signal transduction pathway. The AtDREB1A transcription factor in Arabidopsis induces all the coldregulated genes possessing a $\mathrm{C}$ repeat (CRT) or dehydration responsive elements in their promoter regions. Transgenic tomato plants with an inducible expression of the AtDREB1A gene developed earlier in our laboratory have been reported to be more tolerant to drought stress than the respective wild-type (Rai et al. 2013). The objective of the present study was to investigate the mechanisms how AtDREB1A confers cold stress tolerance to transgenic tomato.

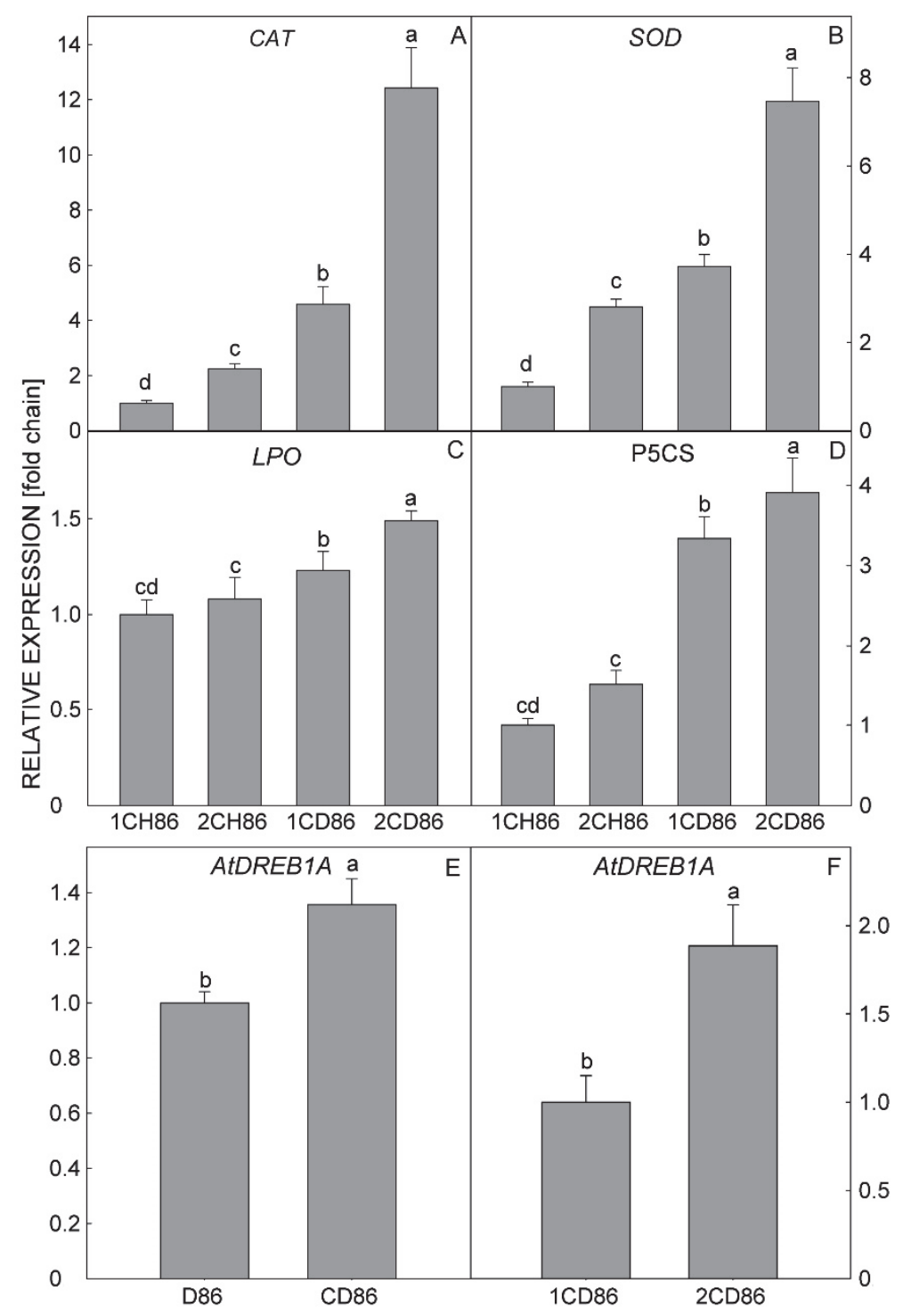

Fig. 5. Effect of subsequent cold stress $\left(4^{\circ} \mathrm{C}\right.$ for $\left.30 \mathrm{~min}\right)$ given to 60 -d-old plants on expression of $C A T(A), S O D(B), L P O(C)$, P5CS (D), and AtDREB1A (E,F) genes in wild-type (H86) and transgenic (D86) plants where "1" represents plants which experienced the cold stress for the first time and "2" represents plants which experienced the second cold stress. Means \pm SEs, $n=3$, values followed by the same letter are not significantly different $(P>0.05)$ according to the Duncan multiple range test. CH86 - wild type cold treated plants, CD86 - transgenic cold treated plants.

The RWC is directly associated with the adaptability of plants to cold stress, and plants with a higher RWC are often considered as more cold tolerant. In our study, wild- type plants showed a more sensitive response to the low temperature showing a lower RWC and a higher EL than transgenic plants. The cell membrane is usually the 
primary target of many environmental stresses. Chilling stress affects cell membrane integrity and stability due to the excessive production of ROS (Khare et al. 2010). Similar results were earlier reported in the case of transgenic tomato plants expressing $r d 29 A: \because A t C B F 1$ (Singh et al. 2011).

Total chlorophyll content is one among several physiological indices that are used to evaluate the response of plants to stress. Carotenoids protect photosynthetic apparatus from photo-oxidation, which occurs due to excess radiation energy, and also reduce ROS (Heath et al. 2013). Earlier studies have reported that overexpression of $A t C B F 3$ can effectively regulate the expression of several photosynthetic genes (Goulas et al. 2006). Improved chilling tolerance observed in $A t C B F$ expressing transgenic potatoes is connected with dark green leaves due to a higher chlorophyll content and an enhanced photosynthetic potential compared to wild-type plants (Pino et al. 2008). Improved chlorophyll content of AtDREB $1 A$ transgenic tomato plants under the cold stress compared to wild-type plants shows their tolerance to the cold stress.

Induction of cold-responsive genes under lowtemperature stress leads to the production of compatible osmolytes and proteins in a plant, which helps to acclimate to a stress (Zhu 2001). Under stress, various osmoprotectants, like proline, glycine-betaine, mannitol, polyamines, etc., accumulate not only to adjust an osmotic balance but also to remove toxic free radicals and to protect the structure of macromolecules and cellular membranes (Hoekstra et al. 2001). Proline is one of the major compatible osmolytes in cells and plays a vital role in protecting proteins and membranes from damaging effects of ROS and, therefore, it is an indicator of plant response to environmental stress. An elevated content of proline under cold stress was reported in transgenic tomato plants overexpressing AtDREB1B/CBF1 (Hsieh et al. 2002, Singh et al. 2011). These plants also have an elevated expression of the key proline biosynthetic gene P5CS. Transgenic plants over-expressing the P5CSF $129 \mathrm{~A}$ gene show a four times higher proline content than nontransgenic plants (Dibax et al. 2010). Our findings are consistent with earlier reports showing the role of $D R E B S / C B F s$ in providing tolerance to freezing stress by targeting multiple cold-responsive genes and by accumulation of cellular proline (Jaglo-Ottosen et al. 1998).

A defense system in plants against a low-temperature stress is strengthened by the accumulation of compatible solutes like TSS. Particularly sucrose, raffinose, glucose, and fructose protect plants in several ways as an osmoprotectant, the source of nutrients, the controller of plant metabolism, and also the protectors of cell membranes (Shao et al. 2006). Although the change in TSS was lower in transgenic plants than in wild-type plants, the overall TSS content in transgenic plants was significantly higher under the cold stress, making the plants more stress tolerant. The results are in parallel to earlier studies in $A t C B F 3$ over-expressing transgenic tomato (Hsieh et al. 2002), where chilling stress significantly enhances accumulation of TSS in transgenic plants.

Malondialdehyde is an end product of decomposition of polyunsaturated fatty acids in phospho-lipids, and it is an indicator of the degree of cell injury (Song et al. 2011). Thus, the lower accumulation of malondialdehyde in transgenic plants was the indicator of a reduced membrane damage under the cold stress. A low content of malondialdehyde has been earlier correlated with a significant cold stress tolerance in transgenic tomatoe over-expressing the SlGMEs gene (Zhang et al. 2011). Exposure to cold stress increases the content of superoxide and hydroxyl free radicals, which cause an increased membrane lipid peroxidation leading to membrane injury. An increase in the amount of leaf $\mathrm{H}_{2} \mathrm{O}_{2}$ under a low-temperature stress has been previously reported in several crops (Prasad et al. 1994). Transgenic plants efficiently reduce the accumulation of $\mathrm{H}_{2} \mathrm{O}_{2}$ under stress conditions. This could be an outcome of elimination of superoxide and $\mathrm{H}_{2} \mathrm{O}_{2}$ production or efficient scavenging by antioxidant enzymes (Baek and Skinner 2003). The higher activities of SOD and CAT under the cold stress have been detected in tomato transgenic plants together with the lower of $\mathrm{H}_{2} \mathrm{O}_{2}$ content. Even under optimal growth conditions, the activities of SOD and CAT were higher in transgenic plants than in wild-type plants. Our results support a previous report where overexpression of the $C B F 1$ gene induces the upregulation of antioxidant enzymes (Hsieh et al. 2002). Non-enzymatic water-soluble antioxidant AsA is highly synthesized in plants under adverse stress conditions and plays a key role in scavenging ROS (Zhang et al. 2011). An increase in total AsA content in transgenic plants helps them scavenge ROS.

Transgenic plants were better adapted to cold stress, which can be seen from a slight increase in root length and shoot length. Transgenic plants retained more water and have a lower dry/fresh mass ratio than wild-type plants. Plant defense against cold stress begins at the molecular level by inducing the expression of stressresponsive genes that further brings about a series of biochemical, physiological, and morphological changes at the cellular level ultimately resulting in acclimation of plants to cold stress. As discussed earlier, proline is an important osmoprotectant and synthesis of proline enhances under cold stress to maintain an osmotic balance. The P5CS is one of the main proline biosynthetic pathway genes and its expression is induced by cold stress (Amini et al. 2015). The expression of P5CS and other antioxidant genes also correlated with the enhanced accumulation of their products like proline, SOD, and CAT. The results indicate the adaptation of transgenic plants to chilling stress by the significant induction of antioxidant gene expressions.

The memory of a plant is characterized by an enhanced or more efficient or more rapid response to subsequent stress (Crisp et al. 2016). Although the exact mechanism of stress memory in plants is not clear at 
present, several factors, like an accumulation of signaling compounds, protective metabolites, transcription factors, and epigenetic modifications, were shown to play a major role (Fleta-Soriano and Munne-Bosch 2016). We also showed that the transgenic plants expressing the AtDREB1A gene were superior in memorizing the cold stress compared to wild-type plants. It could be due to the accumulation of the AtDREB1A transcription factor and/or signaling compounds induced by the first cold stress. The available AtDREB1A transcription factor could quickly respond to the subsequent cold stress in an effective way by activating downstream cold regulated genes. The cold stress tolerant plants can be developed by simply exposing them to chilling temperatures at the seedling stage for a specified period termed as priming or hardening. The temperature and the duration of exposure need to be standardized for individual crops.

\section{References}

Aebi, H.: Catalase in vitro. - Methods Enzymol. 105: 121-126, 1984.

Akhtar, M., Jaiswal, A., Taj, G., Jaiswal, J.P., Qureshi, M.I., Singh, N.K.: $D R E B 1 / C B F$ transcription factors: their structure, function and role in abiotic stress tolerance in plants. - J. Genet. 91: 385-395, 2012.

Amini, S., Ghobadi, C., Yamchi, A.: Proline accumulation and osmotic stress: an overview of P5CS gene in plants. - Mol. Breed. 3: 44-55, 2015.

Baek, K.H., Skinner, D.Z.: Alteration of antioxidant enzyme gene expression during cold acclimation of near-isogenic wheat lines. - Plant Sci. 165: 1221-1227, 2003.

Bates, L.S., Walden, R.P., Teare, I.D.: Rapid determination of free proline for water stress studies. - Plant Soil 39: 205207, 1973.

Chaitanya, K.S.K., Naithani, S.C.: Role of superoxide lipid peroxidation and superoxide dismutase in membrane perturbation during loss of viability in seeds of Shorea robusta Gaertn.f. - New Phytol. 126: 623-627, 1994.

Chinnusamy, V., Zhu, J., Zhu, J.K.: Gene regulation during cold acclimation in plants. - Physiol. Plant. 126: 52-61, 2006.

Crisp, P.A., Ganguly, D., Eichte,n S.R., Borevitz, J.O., Pogson, B.J.: Reconsidering plant memory: intersections between stress recovery, RNA turnover, and epigenetics. - Sci. Adv. 2: $1501340,2016$.

Dibax, R., Deschamps, C., Bespalhok-Filho, J.C., Vieira, L.G.E., Molinari, H.B.C., De Campos, M.K.F., Quoirin, M.: Organogenesis and Agrobacterium tumifaciens-mediated transformation of Eucalyptus saligna with P5CS gene. Biol. Plant. 54: 6-12, 2010.

DuBois, M., Gilles, K.A., Hamilton J.K., Rebers, P.A., Smith, F.: Colorimetric method for determination of sugars and related substances. - Anal. Chem. 28: 350-356, 1956.

Fedoroff, N.V., Battisti, D.S., Beachy, R.N. , Cooper, P.J., Fischhoff, D.A., Hodges, C.N., Knauf, V.C., Lobell, D., Mazur, B.J., Molden, D., Reynolds, M.P., Ronald, P.C., Rosegrant, M.W., Sanchez, P.A., Vonshak, A., Zhu, J.K.: Radically rethinking agriculture for the $21^{\text {st }}$ century. Science 327: 833-834, 2010.

Fleta-Soriano, E., Munné-Bosch, S.: Stress memory and the inevitable effects of drought: a physiological perspective. Front. Plant. Sci. 7: 143, 2016.
In conclusion, the transgenic tomato plants with the induced expression of $A t D R E B 1 A$ under the control of the $r d 29 A$ promoter showed better growth under the chilling stress $\left(4{ }^{\circ} \mathrm{C}\right.$ for $\left.5 \mathrm{~d}\right)$ in comparison to wild-type plants. The transgenic plants had a higher RWC, amount of osmolytes, activities of antioxidant enzymes, and a lower EL and ROS accumulation compared to wild-type plants. The transgenic plants also showed a slightly lower reduction in chlorophyll content than wild-type plants. The expression of P5CS, a major gene of proline biosynthetic pathway, and other antioxidant enzyme genes $S O D, C A T$, and $L P O$ were induced in transgenic plants under the cold stress. Moreover, the transgenic plants showed a better stress memory of the previous cold stress and responded more quickly and efficiently to the subsequent cold stress in comparison to wild-type tomato plants.

Gilmour, S.J., Zarka, D.G., Stockinger, E.J., Salazar, M.P., Houghton, J.M., Thomashow, M.F.: Low temperature regulation of the Arabidopsis $\mathrm{CBF}$ family of AP2 transcriptional activators as an early step in cold-induced COR gene expression. - Plant J. 16: 433-442, 1998.

Goulas, E., Schubert, M., Kieselbach, T., Kleczkowski, L.A., Gardestrom, P., Schroder, W., Hurry, V.: The chloroplast lumen and stromal proteomes of Arabidopsis thaliana show differential sensitivity to short- and long-term exposure to low temperature. - Plant J. 47: 720-734, 2006.

Heath, J.J., Cipollini, D.F., Stireman, J.O.: The role of carotenoids and their derivatives in mediating interactions between insects and their environment. - Arthropod Plant Interact. 7: 1-20, 2013.

Heath, R.L., Packer, L.:Photoperoxidation in isolated chloroplasts: I. Kinetics and stechiometry of fatty acid peroxidation. - Arch. Biochem. Biophys. 125: 189-198, 1968.

Hoekstra, F.A., Golovina, E.A., Buitink, J.: Mechanism of plant desiccation tolerance. - Trends Plant Sci.6: 1360-1385, 2001.

Hsieh, T.H., Lee, J.T., Yang, P.T., Chiu, L.H., Charng, Y.Y., Wang, Y.C., Chan, M.T.: Heterology expression of the Arabidopsis C-repeat/dehydration response element binding factor 1 gene confers elevated tolerance to chilling and oxidative stresses in transgenic tomato. - Plant Physiol. 129: 1086-1094, 2002.

Jaglo-Ottosen, K.R., Gilmour, S.J., Zarka, D.G., Schabenberger, O., Thomashow, M.F.: Arabidopsis CBF1 overexpression induces $C O R$ genes and enhances freezing tolerance. Science 280: 104-106, 1998.

Jana, S., Choudhury, M.A.:Glycolate metabolism of three submerged aquatic angiosperm during aging. - Aquat. Bot. 12: 345-354, 1981.

Janska, A., Aprile, A., Zamecník, J., Cattivelli, L., Ovesna, J.: Transcriptional responses of winter barley to cold indicate nucleosome remodelling as a specific feature of crown tissues. - Funct. integr.Genomics 11: 307-325, 2011.

Khare, N., Goyary, D., Singh, N.K., Shah, P., Rathore, M., Anandhan, S., Sharma, D., Arif, M., Ahmed, Z.: Transgenic tomato cv. PusaUphar expressing a bacterial mannitol-1phosphate dehydrogenase gene confers abiotic stress 
tolerance. - Plant Cell Tissue Organ Cult. 103: 267-277, 2010.

Law, M.Y., Charles, S.A., Halliwell, B.: Glutathione and ascorbic acid in spinach (Spinaceaoleracea) chloroplast: the effect of hydrogen peroxide and paraquat. - Biochem. J. 210: 899-903, 1983.

Lichtenthaler, H.K., Buschmann, B.C.: Current Protocols in Food Analytical Chemistry. - John Wiley and Sons, New York 2001.

Liu, X.Q., Liu, C.Y., Guo, Q., Zhang, M., Cao, B.N., Xiang, Z.H., Zhao, A.C.: Mulberry transcription factor MnDREB4A confers tolerance to multiple abiotic stresses in transgenic tobacco. - PLoS ONE 22: 0145619, 2015.

Livak, K.J., Schmittgen, T.D.: Analysis of relative gene expression data using real time quantitative PCR and the 2$\triangle \Delta C T$ method. - Methods 25: 402-408, 2001.

Mishra, H.P., Fridovich, I.: The role of superoxide anion in the auto-oxidation of epinephrine and a simple assay for superoxide dismutase. - J. biol. Chem. 247: 3170-3175, 1972.

Pino, M.T., Skinner, J.S., Jeknic, Z., Hayes, P.M., Soeldner, A.H., Thomashow, M.F., Chen, T.H.H.: Ectopic AtCBF1 over-expression enhances freezing tolerance and induces cold acclimation-associated physiological modifications in potato. - Plant Cell Environ. 31: 393-406, 2008.

Prasad, T.K., Anderson, M.D., Martin, B.A., Stewart, C.R.: Evidence for chilling-induced oxidative stress in maize seedlings and a regulatory role for hydrogen peroxide. Plant Cell 6: 65-74, 1994.

Rai, G.K., Rai, N.P., Kumar, S., Yadav, A., Rathaur, S., Singh, M.: Effects of explant age, germination medium, pre-culture parameters, inoculation medium, $\mathrm{pH}$, washing medium, and selection regime on Agrobacterium-mediated transformation of tomato. - In Vitro cell. dev. Biol. Plant. 48:565-78, 2012.

Rai, G.K., Rai, N.P., Rathaur, S., Kumar, S., Singh, M.: Expression of $r d 29 A:: A t D R E B 1 A / C B F 3$ in tomato alleviates drought-induced oxidative stress by regulating key enzymatic and non-enzymatic antioxidants. - Plant Physiol. Biochem. 69: 90-100, 2013.

Rozen, S., Skaletsky, H.J.: Primer3, http://www-genome. wi.mit.edu/genome_software/other/primer3.html, 1998.
Shao, H.B., Chen, X.Y., Chu, L.Y., Zhao, X.N., Wu, G., Yuan, Y.B., Zhao, C.X., Hu, Z.M.: Investigation on the relationship of proline with wheat anti-drought under soil water deficits. - Colloids Surf. B. Biointerfaces 53: 113-119, 2006.

Singh, S., Rathore, M., Goyary, D., Singh, R.K., Anandhan, S., Sharma, D.K., Ahmed, Z.: Induced ectopic expression of $A t-C B F 1$ in marker-free transgenic tomatoes confers enhanced chilling tolerance. - Plant Cell Rep. 30: 10191028, 2011.

Song, S.Y., Chen, Y., Chen, J., Dai, X.Y., Zhan, W.H.: Physiological mechanisms underlying OsNAC5-dependent tolerance of rice plants to abiotic stress. - Planta 234: 331345, 2011.

Weiss, J., Egea-Cortines, M. (only two authors): Transcriptomic analysis of cold response in tomato fruits identifies dehydrin as a marker of cold stress. - J. appl. Genet. 50: 311-319, 2009.

Wibowo, A., Becker, C., Marconi, G., Durr, J., Price, J., Hagmann, J., Papareddy, R., Putra, H., Kageyama, J., Becker, J., Weigel, D.: Hyperosmotic stress memory in Arabidopsis is mediated by distinct epigenetically labile sites in the genome and is restricted in the male germline by DNA glycosylase activity. - Elife 5: e13546, 2016.

Yamaguchi-Shinozak,i K., Shinozaki, K.: Transcriptional regulatory networks in cellular responses and tolerance to dehydration and cold stresses. - Annu. Rev. Plant Biol. 57: 781-803, 2006.

Zhang, C.J., Liu, J.X., Zhang, Y.Y., Cai, X.F., Gong, P.J., Zhang, J.H., Wang, T.T., Li, H.X., Ye, Z.B.: Overexpression of SlGMEs leads to ascorbate accumulation with enhanced oxidative stress, cold, and salt tolerance in tomato. - Plant Cell Rep. 30: 389-398, 2011.

Zhu, J.K.:Cell signalling under salt, water and cold stresses. Curr. Opin. Plant. Biol. 4: 401-406, 2011.

Zong, J.M., Li, X.W., Zhou, Y.H., Wang, F.W., Wang, N., Dong, Y.Y., Yuan, Y.X., Chen, H., Liu, X.M., Yao, N., Li, H.Y.: The AaDREBltranscription factor from the coldtolerant plant Adonis amurensis enhances abiotic stress tolerance in transgenic plant. - Int. J. mol. Sci. 17: 611-626, 2016. 